This item was submitted to Loughborough's Research Repository by the author.

Items in Figshare are protected by copyright, with all rights reserved, unless otherwise indicated.

\title{
In vitro localization of intracranial haematoma using electrical impedance tomography semi-array
}

PLEASE CITE THE PUBLISHED VERSION

http://dx.doi.org/10.1016/j.medengphy.2014.10.001

\section{PUBLISHER}

Elsevier / ( I Institute of Physics and Engineering in Medicine (IPEM)

VERSION

AM (Accepted Manuscript)

\section{PUBLISHER STATEMENT}

This work is made available according to the conditions of the Creative Commons Attribution-NonCommercialNoDerivatives 4.0 International (CC BY-NC-ND 4.0) licence. Full details of this licence are available at: https://creativecommons.org/licenses/by-nc-nd/4.0/

\section{LICENCE}

CC BY-NC-ND 4.0

\section{REPOSITORY RECORD}

Ayati, S. Bentolhoda, Kaddour Bouazza-Marouf, and David Kerr. 2019. "In Vitro Localization of Intracranial Haematoma Using Electrical Impedance Tomography Semi-array”. figshare.

https://hdl.handle.net/2134/16582. 


\section{In vitro localisation of intracranial haematoma using electrical 2 impedance tomography semi-array}

3 S. Bentolhoda Ayati, Kaddour Bouazza-Marouf, David Kerr

4

5

Wolfson School of Mechanical and Manufacturing Engineering, Loughborough University, Loughborough, LE11 $3 T U$, United Kingdom

\section{Abstract}

7 Electrical Impedance Tomography is a non-invasive and portable method that has good 8 potential as an alternative to the conventional modalities for early detection of intracranial 9 haematomas in high risk patients. Early diagnosis can reduce treatment delays and most significantly can impact patient outcomes. Two eight-electrode layouts, a standard ring full array (FA) and a semi-array (SA), were investigated for their ability to detect, localise and quantify simulated intracranial haematomas in vitro on ovine models for the purpose of early diagnosis. SA layout speeds up electrode application and avoids the need to move and lift the patient's head. Haematomas were simulated using gel samples with the same conductivity as blood. Both layouts, FA and SA, could detect the presence of haematomas at any location within the skull. The mean of the relative radial position error with respect to the brain radius was $7 \%$ for $\mathrm{FA}$ and $6 \%$ for $\mathrm{SA}$, for haematomas close to the electrodes, and $11 \%$ for SA for haematomas far from the electrodes at the back of the head. Size estimation was not as good; the worst size estimation error for FA being around 30\% while the best for SA was $50 \%$ for simulated haematomas close to the electrodes.

Keywords 


\section{Introduction}

24 Head injury is the main cause of death among young adults and children and may become the third greatest global death cause by 2020 , due to the substantial number of associated deaths and cases of disability [1]. The UK National Confidential Enquiry into Patient, Outcome and Death (NCEPOD) reported that more than half of patients that required neurosurgical advice were taken to hospitals with no on-site neurosurgical provision and only $14 \%$ of patients requiring secondary transfer to a neurosurgical centre had access to neurosurgical treatment within four hours [2]. Patients treated in a non-neurosurgical centre had a $26 \%$ increase in mortality and a 2.15 fold increase in the risk of death compared to patients treated at a neurosurgical centre [3]. First responders need more information on the neurological condition of their patient. In particular, they require information on potentially evolving haematomas which may need prompt and rapid action. This information is vital for proper triage, and to ensure the best possible decisions are made for the patient's welfare.

Haematomas expand and increase the intracranial pressure on the brain. A growing haematoma will cause severe and even permanent damage to the delicate tissue of the brain, morbidity, and eventual death of the patient [4]. Haematomas are classified based on their location. Epidural Haematomas form between the skull and the dura-mater. They occur because of trauma and a tear in an artery, often to the temple, where the middle meningeal artery is located. Subdural haematomas occur because of trauma and a tear in veins beneath the dura-mater in the brain. A subdural haematoma is very close to the brain and may cause a serious problem. Intracerebral Haematomas, occur within the brain parenchyma itself due to bleeding from trauma or uncontrolled high blood pressure. The development of the haematoma from benign to symptomatic can be sudden, and a patient can change from lucid 
47 known that the time from injury-to-diagnosis-to-treatment is a key factor in patient outcome, and must be minimised for a patient to make a full recovery.

Electrical Impedance Tomography (EIT) reconstructs cross-sectional images of the conductivity distribution of the internal components of the brain, based on non-invasive voltage measurements through an array of electrodes on its boundary. Blood has a high electrical conductivity contrast relative to other cranial tissues and thus its appearance can be detected and monitored using EIT [6].

Head injuries and haematoma are often accompanied by other traumatic injuries that can be aggravated by unnecessary movement, including the placing of electrodes around the head. Therefore, it is desirable to develop methods that do not involve applying electrodes at the back of the head. Placement of the electrodes on the anterior of the head avoids exacerbating existing injuries and removes the need to lift the patient. However, good localization of a haematoma is hampered by eliminating the electrodes at the back of the head. To conquer the quality reduction of the images and to minimize these errors, several reconstruction strategies have been proposed previously [7]. The purpose of this study is to evaluate the performance of EIT, using optimised eight-electrode configurations. This includes an investigation of the different configurations to evaluate their ability to detect and localise anomalies similar to haematoma in the human head, for the purpose of early diagnosis. Using the minimum number of electrodes is always desirable in clinical applications since it may also speed up the electrode setup process in emergency cases. The proposed electrode configurations are evaluated for the detection and localisation of simulated haematomas in vitro using an ovine model. Intracerebral haematoma detection has been considered in previous studies using EIT [8]. Epidural and subdural haematomas are considered in this study since their location can represent the worst case with respect to the SA configuration. 


\section{Methods}

In EIT, the process is divided into a forward problem and an inverse problem. To reconstruct the conductivity distribution images through the EIT inverse problem the forward problem on a prototype model has to be solved. For general cases, a numerical method such as finite element analysis is required to implement the model and solve the forward problem. Initially, a simple forward model based on a circular shape with a homogenous conductivity distribution may be used to calculate the sensitivity matrices [9]. Better results are obtained if the forward model exactly matches the object in terms of internal conductivity distribution and external geometry. In principle, an incorrect estimate of boundary shape will introduce artefacts and reduce the quality of the reconstructed images. However, more realistic models need to be used carefully since inaccurate prior information may yield images worse than those reconstructed with a simple forward model [10]. In practice, it is difficult to specify an accurate model for an individual head because head geometry varies from patient to patient.

2.1.Full and semi-array electrode layouts

In this study, two eight-electrode layout strategies were investigated in vitro and compared in terms of their ability to detect and localise intracranial haematomas. The first one was a standard ring layout or full-array (Figure 1a), where the eight electrodes were placed equally spaced around the head. The second layout was a novel electrode configuration applied to the front of the head. This so-called semi-array (SA) configuration consisted of a set of eight electrodes separated by angle of $36^{\circ}$ in a semi-circular profile (Figure 1b). This layout simplifies the application of the electrodes and avoids the need to move and lift the patient's head. An adjacent current pattern was applied to both layouts, wherein current was applied in turn to pairs of adjacent electrodes, and voltages were measured across other pairs of adjacent electrodes. In the SA, use of this scheme included measurements and current applications 
between the last-numbered and first electrodes positioned at the end of the array and approximately $108^{\circ}$ apart. Both layouts involved 8 current positions and a total of 40 voltage measurements. Experiments were performed on an ovine model using both layouts and the results obtained from the SA layout were compared with data from the standard eightelectrode full-array (FA) layout to determine the ability of the SA to detect and localise intracranial haematomas. Restricting electrodes at the back of the head limits the resolution and thus inferior localisation of the anomaly can be expected, compared to that of the FA layout.

\subsection{Data generation}

In vitro ovine experiments were performed in conjunction with an eight-electrode EIT system to determine the potential of this configuration to provide good results in vivo. To obtain the experimental measurements, a prototype 16-electrode EIT system known as the "EITLboro" rig was used. The structure of this device is presented in Figure 2. The system is controlled by a microcontroller connected to a PC through a serial port. A graphical user interface was developed using Visual Basic (VB). A sinusoidal current generated by a constant current source was injected through one pair of adjacent electrodes and the corresponding boundary potentials were measured over pairs of the remainder of the neighbouring electrodes using a multiplexer. The input pair of electrodes was switched over all adjacent electrodes pairs and the measurement procedure was repeated for all possible adjacent pairs. The performance of this system was previously evaluated using phantom experiments [11]. The results showed a high level of accuracy with an average accuracy of $93.5 \%$ for the system. This EIT system has 16 channels and operates with a temporal resolution of 100 frames per second. For this experiment, a constant current of $1 \mathrm{~mA}$ at a frequency of $50 \mathrm{kHz}$ and 8 electrodes were chosen. 
Five freshly skinned sheep heads (labelled as A, B, C, D and E) were obtained from a local butcher. The locations of the 8 electrodes for each layout were marked in different colours on the skull. Equal distance between electrodes has been considered around the head for the FA and in the anterior of the head for the SA according to the perimeter measurement of each head. Eight $\mathrm{Ag} / \mathrm{AgCl}$ disk electrodes (Unimed Electrode Supplies Ltd) were fastened to the skull using conductive paste (Unimed Electrode Supplies Ltd) for the FA layout (Figure 3a). These electrodes were also soldered to the wires to connect to the skull on the interior of the head using conductive paste for the SA layout (Figure 3b).

A saline solution with the same conductivity as blood $(0.67 \mathrm{~S} / \mathrm{m})$ was made with a concentration of $0.33 \%$ [weight/volume] of sodium chloride in water. In order to localise haematoma in vitro, the position of the anomaly has to be known with a good estimation. To simulate a more realistic haematoma in an accurate location, the saline solution was transformed to gel. The saline solution was stirred using a magnetic stir bar at a temperature of $70^{\circ} \mathrm{C}$ while agar powder was added to achieve the desired gel concentration $(1.9 \%$ by weight). Then the solution was poured into a $1 \mathrm{~cm}$ diameter tube and allowed to cool at room temperature. The gel sample was removed from the tube and cut into one tenth of the diameter of each brain to simulate pockets of blood. The conductivity of the gel sample was measured and found to be the same as the conductivity of the saline solution. A 2-terminal measurement was performed to measure the gel conductivity and the same gel samples were used on each subject. An AC voltage was applied across the gel at a frequency of $50 \mathrm{kHz}$ at room temperature using a waveform function generator connected in series with a digital multimeter to measure the AC current and voltage across the gel. The circuit was calibrated with multiple known resistances, and the conductivity measurements were compared to published data [12]. 
143 All the skulls were cut in approximately half using a bone saw. The top half of the skull was 144 carefully removed and the brain was exposed in order to position the anomalies (Figure 4).

145 Gel samples were placed superficially on top of the brain lobe and the top half of the skull 146 was replaced. The anomaly was located in different positions along the $\alpha, \alpha \beta, \beta, \beta \gamma$ and $\gamma$ axes $147\left(\right.$ at $\theta=0^{\circ}, 45^{\circ}, 90^{\circ}, 135^{\circ}$ and $180^{\circ}$ ), with the anomaly centre placed successively at a relative radial displacement of 0.8 from the brain centre. Five locations were considered in total as shown in Figure 1 and the measurements performed using both layouts for each anomaly to study reconstruction, detection and localisation characteristics. The aim was to study and compare the ability of the SA and FA layouts to detect and localise these anomalies, especially for the SA, and to evaluate the dependency of the results on the distance of the anomaly from the electrodes.

\subsection{Reconstruction}

In this study, EIT difference images were reconstructed based on the assumption that the conductivity changes are small enough. The relationship between the boundary voltage measurement changes and internal conductivity changes can be expressed with a sensitivity matrix (S) as in Eq. (1). S was calculated from forward solutions of a two-dimensional disk finite element model with a homogenous conductivity distribution [9].

161 Conductivity changes $(\Delta \sigma)$ can be determined by inverting the sensitivity matrix; however, $S$ 162 is ill-conditioned and not square. Since the EIT inverse problem is severely ill-posed and a small amount of noise on boundary measurements, $\Delta \mathrm{V}$, can cause a large oscillation for the solution, a regularization technique was used to reduce this effect by improving the condition of S [6].The Truncated Singular Value Decomposition (TSVD) method which has previously been identified as a suitable regularization method [13] was used to regularize the inversion 
of the sensitivity matrix. The truncation point $\mathrm{k}$ needs to be chosen carefully, less than or equal to the rank of the matrix, as it would otherwise produce inaccurate images. The truncation point was chosen depending on the noise level in the voltage measurements and

170 the rank of the sensitivity matrix on inspection of the L-curve of experimental data [14]. The

171 truncation numbers were almost the same for all the datasets. The pseudo-inversion $\left(\mathrm{S}^{\dagger}\right)$ was

172 achieved using TSVD and images were obtained using Eq. (2).

$173 \Delta \sigma=\mathrm{S}^{\dagger} \Delta \mathrm{V}$

174 In the SA, measurement sensitivity depends strongly on the anomaly location since the electrodes are not placed all over the head. Some reconstructed anomalies located far from the electrodes in the posterior region were almost invisible or erroneous when TSVD

177 reconstruction was used. Therefore, in order to enhance image reconstruction quality and 178 improve anomaly localisation, the sensitivity matrix was weighted with a diagonal matrix composed of a system blurring property, which was directly calculated from the sensitivity matrix [7]. In the Weighted Pseudo-Inverse (WPI) method, reconstruction was weighted with $\mathrm{P}$ prior to pseudo-inversion. The entries of a diagonal weighting matrix $(\mathrm{P})$ were calculated using Eq. (3) where ne is the total number of elements. The blur matrix (B) is dimensionless and can be pre-calculated from the sensitivity matrix $S$ via Eq. (4). Then the reconstruction was modified to obtain images using Eq. (5).

$p_{j}=\left(\sum_{i=1}^{n e} B_{i j}^{2}\right)^{-1}$

185

$B=\mathrm{S}^{\dagger} \mathrm{S}$ 
189 The position of the anomaly (x,y) can be estimated from the reconstructed images by 190 averaging the positions of all elements, weighted by their conductivity changes [8] via Eqs. (6) 191 and (7):

$$
\begin{aligned}
& \mathrm{x}=\frac{\sum_{i=1}^{n e} \Delta \sigma_{i} a_{i} x_{i}}{\sum_{i=1}^{n e} \Delta \sigma_{i} a_{i}} \\
& \mathrm{y}=\frac{\sum_{i=1}^{n e} \Delta \sigma_{i} a_{i} y_{i}}{\sum_{i=1}^{n e} \Delta \sigma_{i} a_{i}}
\end{aligned}
$$

192 The anomaly location $(\mathrm{x}, \mathrm{y})$ is effectively the centre of the reconstructed anomaly. The values $193 x_{i}$ and $y_{i}$ are the coordinates of the centre and $a_{i}$ is the area of each element. The difference between the actual position of the anomaly and its reconstructed location within the xy plane can be calculated as the absolute location errors (relative radial error, $D_{x y}$ ) quoted as a 196 fraction of the brain radius, $\mathrm{R},[8]$ via Eq. (8):

$D_{x y}=\frac{\sqrt{(\Delta x)^{2}+(\Delta y)^{2}}}{R}$

\subsection{Quantification}

The anomaly size was assessed with a characteristic parameter, the quantity index (QI), defined in Eq. 9 as an EIT image parameter that correlates with the anomaly size [15]. The quantity index is the sum of conductivity change multiplied by the area of the element over 201 the image area:

$$
Q I=\sum_{i=1}^{n e} \Delta \sigma_{i} a_{i}
$$

where, for an element (or pixel) $i, \Delta \sigma_{i}$ is the conductivity change reconstructed in the $i$-th element. QI values should be constant since the anomaly sizes are the same during the experiment over all the positions. 
The data for intracranial haematomas in five ovine models were successfully collected with the EITLboro rig and used to reconstruct the images. To improve the SNR of EIT, a sequence of at least 100 frames of data was collected before the anomalies were introduced. These were averaged and used as the reference data set. For each anomaly position, 100 frames of data were collected and averaged as the perturbation data for that anomaly position.

\subsection{Comparing FA and SA localisation}

212 The simulated haematomas were localised using the reconstructed images. The results of the anomaly localisation in the five ovine models using both FA and SA layouts are presented in Figures 5 and 6 respectively. The position of the simulated haematomas varied as a function of angle from $0^{\circ}$ to $180^{\circ}$ with $45^{\circ}$ increments at relative radial displacement of 0.8 from the brain centre. Locations were normalized to a circular shape with unit diameter. The discrepancy between the actual and reconstructed locations is illustrated by arrows. For illustration, reconstructed images of the anomalies in subject E using FA configuration are shown in Figure 5 and reconstructed images of the anomalies in subject $\mathrm{C}$ using SA configuration are shown in Figure 6. The simulated anomalies were detected for all the positions using both layouts; however FA results were in general superior to the SA results. Relative radial localisation errors for five ovine models using FA and SA are shown in Figures 7 and 8 respectively. As expected, the SA layout localised simulated anomalies as well as the FA layout apart from the anomalies placed far away from the electrodes. Localisation errors were larger for reconstructions of the anomalies at $135^{\circ}$ with the worst value of $D_{x y}$ being 0.3265 using the SA layout, and 0.0828 using the FA layout, both values being with respect to unit radius. The mean and variance of the relative radial errors are presented in table 1. Both the FA and SA electrode layouts could localise simulated 
haematomas well, producing a maximum mean relative radial error of 0.0714 and 0.2364 respectively at $135^{\circ}$ with respect to the unit radius.

\subsection{Comparing FA and SA size estimation}

The reconstructed images of the simulated haematomas were gathered and post-processed to determine QI values. The ability of the FA and SA layouts to quantify these anomaly sizes was compared to the actual QI. The normalized QI values for five ovine models using the FA electrode layout over the image plane against anomaly position for five angles are shown in Figure 9. For the SA layout, the same positions of anomalies in directions of $\alpha, \alpha \beta, \beta, \beta \gamma$ and $\gamma$ were used. Normalized QI values from the SA reconstructions of simulated anomalies are shown in Figure 10. Size estimation results for the FA layout are superior to those for the SA layout. The worst QI error in reconstructions using the FA layout was around 30\%. According to the reconstructed images, the size of the simulated anomalies at $0^{\circ}$ and $45^{\circ}(\alpha$ and $\alpha \beta$ ) are measured by maximum error of approximately $55 \%$ and $50 \%$ respectively in the case of the SA electrode layout. For simulated anomalies placed at $90^{\circ}(\beta)$ using the SA layout, although in one case the QI value is large, the worst QI error in the others is about $55 \%$. The SA layout performance to quantify the simulated anomalies at $135^{\circ}$ and $180^{\circ}(\beta \gamma$ and $\gamma$ ) is very poor as in the worst case the anomaly sizes were measured almost three times bigger than their actual size.

\section{Discussion}

4.1.Comparison between full array and semi-array layouts

For the first time, animal studies were performed using EIT to detect and localize haematomas within the skull in an ovine model. In five sheep heads, haematomas were simulated by placing gel samples with the same conductivity as blood at different positions. Two eight-electrode layouts were applied to compare their ability to localise and quantify the 
simulated haematomas. An optimised, novel electrode layout named semi-array (SA) was

254

255

256

257 introduced and its performance was evaluated and compared in vitro with a conventional full array electrode layout. The FA layout performed well in both localisation and size estimation of the anomalies. We believe that the errors in the FA experiments are due to the presence of random and systematic experimental noise. As expected, the SA layout performed well in detecting and localising anomalies close to the electrodes, but slightly worse for anomalies far away from the electrodes. Reduction of the electrodes at the posterior of the head reduced overall image quality and increased uncertainty in estimations of location and size of the anomalies. Large spatial variance and therefore the variability in size estimates of an anomaly because of the restricted number of electrodes at the posterior of the head are inevitable. We believe that the large size estimation errors may have been caused by the noise-generated artefacts in reconstructions and the electrode positions.

\subsection{Anomaly size estimation}

Although QI values depend on the size of the anomaly, the regularisation method and the sensitivity matrix calculation also affect the size estimation. The FA and SA electrode layouts produce different sensitivities in the region at the back of the head and therefore have different QI accuracy. Large size errors in SA may have been related to the smaller sensitivity at the posterior region of the head, far from the electrodes, combined with measurement noise. However, spatial variation of QI was improved by using blurring properties calculated directly from the sensitivity matrix. The truncation number for each case was chosen based on the noise level in the voltage measurements and the rank of the sensitivity matrix on inspection of the L-curve of experimental data.

\subsection{Comparing results with earlier studies}

Using EIT for clinical applications to detect and image bleeding in an animal model has been proven in previous studies $[16,17]$. A group based at the University of Florida has applied 

EIT to detect intra-ventricular haemorrhage (IVH) for neonate applications [8]. In their phantom experimental studies, data were collected by a FA layout with 16 electrodes equally spaced around the head, using adjacent current patterns. Their results showed a maximum radial error of 0.1 and QI error of $30 \%$ which is better than our size estimation accuracy. Sadleir et al. [6] introduced a hemi-array electrode layout for the application of abdominal trauma. QI values of their phantom experimental studies showed a variation of around a factor of 4 , the maximum being $220 \%$. Hemi-arrays have been used in vivo to quantify accumulating abdominal fluid [18] and to monitor lung resistivity by Zlochiver et al [19]. However, the hemi-array electrode layout failed in our application to detect simulated haematomas using ovine models. We believe that the presence of the skull and the geometry structure of the head may restrict the use of the hemi-array in this application. However, our optimised SA electrode layout has shown its reliability to detect, localise and quantify the simulated haematomas in this application. According to earlier studies [20, 21], the quality of the images and consequently the localisation and size errors may be improved by increasing the number of electrodes. However, the objective of this concept is to optimize the number and position of the electrodes in order to speed up the electrode setup process and avoid the need to move and lift the patient's head in emergency cases. We believe that using an eight-electrode configuration is more preferable than using 16 or 32 and that the ensuing results are quite acceptable. Although the use of $2 \mathrm{D}$ imaging restricts the $\mathrm{z}$ direction localisation, the overall process is faster and the results are reliable enough for a useful application to emergency cases.

\subsection{Comparing EIT with current modalities}

Haematomas are typically diagnosed by neurological assessment in the emergency room followed by a Computed Tomography (CT) scan. CT scanners are not portable and thus diagnosis cannot be made until the patient is delivered to the hospital. Moreover, CT 
scanning is not always available for 24 hours a day, and in cases of multiple traumas, it may not be possible to scan the patient until they have been adequately stabilised [22]. Although the sensitivity and resolution of Magnetic Resonance Imaging (MRI) is higher than CT, the transporting requirement of ill patients and equipment compatibility restrict use of this method. EIT is a non-invasive, portable, low-cost, operator independent method that has the potential to monitor and measure the progress of internal bleeding. EIT offers a good alternative to the conventional modalities for early detection, localisation and size estimation of haematomas in high risk patients. Early diagnosis can reduce treatment delays, save on costs and waste, and most significantly, positively impact patient outcomes. Treatment delays can thus be mitigated by giving better and earlier information on haematomas at the triage stage.

\section{Conclusion}

315 This study indicates the feasibility of detection, localisation and size estimation of haematomas in vitro with preliminary EIT imaging on ovine models for the purpose of early diagnosis. Two eight-electrode layouts were compared in vitro on their ability to detect, localise and quantify simulated haematomas. As expected, the FA layout was found to be more robust than the SA layout, having an overall better quality on localisation and size estimation of the simulated haematomas. Although using the SA configuration reduces the sensitivity and accuracy of quantity estimations, an optimized electrode layout that does not require the patient to be lifted for its application would be very convenient for emergency applications where the required accuracy is not critical. 


\section{References}

[1] Dinsmore J. Traumatic brain injury: an evidence-based review of management. Continuing Education in Anaesthesia, Critical Care \& Pain Advance Access 2013.

[2] Treasure T. Trauma: Who cares? A report of the National Confidential Enquiry into Patient Outcome and Death (NCEPOD) 2007:19.

[3] Patel H, Bouamra O, Woodford M, King A, Yates D, Lecky F, On behalf of the Trauma Audit and Research Network. Trends in head injury outcome from 1989 to 2003 and the effect of neurosurgical care: an observational study. The Lancet 2005;366(9496):1538-44.

[4] Voss M, Knottenbelt J, Peden M. Patients who reattend after head injury: a high risk group. BMJ 1995;311(7017):1395-98.

[5] Moore K, Dalley A, Agur A. Clinically Oriented Anatomy. Wolters Kluwer Health/Lippincott Williams \& Wilkins 2010:876-77.

[6] Holder D. Electrical impedance tomography methods, history and applications. UK: IOP Publishing 2005.

[7] Sadleir R, Zhang S, Tucker A, Oh S. Imaging and quantification of anomaly volume using an eight-electrode ‘hemiarray’ EIT reconstruction method. Physiological Measurement 2008;29:913-27. [8] Tang T, Oh S, Sadlier R. A robust current pattern for the detection of intraventricular hemorrhage in neonates using electrical impedance tomography. Ann Biomed Eng 2010;38(8):2733-47.

[9] Bagshaw A, Liston A, Bayford R, Tizzard A, Gibson A, Tidswell A, Sparkes M, Dehghani H, Binnie C, Holder D. Electrical impedance tomography of human brain function using reconstruction algorithms based on the finite element method. Neuroimage 2003;20(2):752-64.

[10] Liston A, Bayford R, Holder D. The effect of layers in imaging brain function using electrical impedance tomography. Physiological Measurement, 2004;25:143-58.

[11] Ayati S, Bouazza-Marouf K, Kerr D, O'Toole M. Performance evaluation of a digital electrical impedance tomography system. Proceedings of the IASTED international symposia in imaging and signal processing in health care and technology, Baltimore, USA, 2012:101-5. 
[12] Kandadai M, Raymond J, Shaw G. Comparison of electrical conductivities of various brain phantom gels: Developing a 'brain gel model'. Materials Science and Engineering C 2012;32:26642667.

[13] Oh S, Tang T, Tucker A, Sadleir R. Normalization of a spatially variant image reconstruction problem in electrical impedance tomography using system blurring properties. Physiological Measurement 2009;30(3):275-89.

[14] Hansen P, O'Leary D. The use of the L-curve in the regularization of discrete ill-posed problems. SIAM Journal of Science and Computing 1993;14(6):1487-503.

[15] Sadleir R, Fox R. Quantification of blood volume by electrical impedance tomography using a tissue equivalent phantom. Physiological Measurement 1998;19:501-16.

[16] Xu Canhua, Dai Meng et al. An optimized strategy for real-time haemorrhage monitoring with electrical impedance tomography. Physiological Measurement 2011; 32(5):585-98.

[17] Xu C-H, Wang L et al. Real-time imaging and detection of intracranial haemorrhage by electrical impedance tomography in a piglet model. The Journal of international medical research 2010; 38(5):1596-604.

[18] Tucker A, Ross E, Paugh-Miller J, Sadlier R. In vivo quantification of accumulating abdominal fluid using an electrical impedance tomography hemiarray. Physiological Measurement 2011;32(2):151-65.

[19] Zlochiver S, Arad M, Radai M, Barak-Shinar D, Krief H, Engelman T, Ben-Yehuda R, Adunsky A, Abboud S. A portable bio-impedance system for monitoring lung resistivity. Med Eng Phys 2007;29(1):93-100.

[20] Yvert B, Bertrand O, Thvenet M, Echallier J, Pernier J. A systematic evaluation of the spherical model accuracy in EEG dipole localization. Electroencephalogr Clin Neurophysiol 1997;102:452-59. [21] Taktak A, Record P, Gadd R, Rolfe P. Data recovery from reduced electrode connection in electrical impedance tomography. Med Eng Phys 1996;18(6):519-522.

[22] Wardlaw J, Lewis S, Sandercock P, Ricci S, Spizzichino L, the I. Why do Italian stroke patients receive CT scans earlier than UK patients? Postgrad Med J. 1999;75(879):18-21. 


\section{Captions}
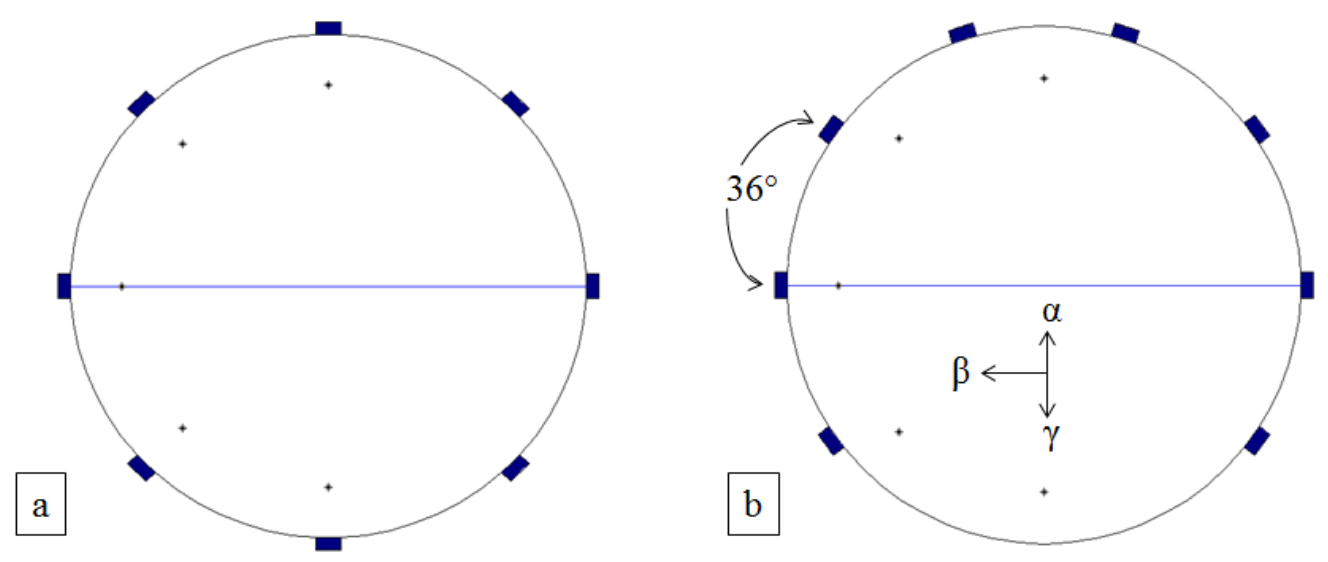

2

3 Figure 1: Electrode positions showing (a) the standard ring layout where the eight electrodes

4 were placed equally spaced around the head, and (b) a novel electrode configuration applied to the

5 front of the head separated by angle of 36 in a semi-circular profile. Stars show the ideal positions

6 of centre of simulated anomalies on sheep's head at relative radius of 0.8 in $\alpha, \alpha \beta, \beta, \beta \gamma$ and $\gamma$ axes

7 directions. 


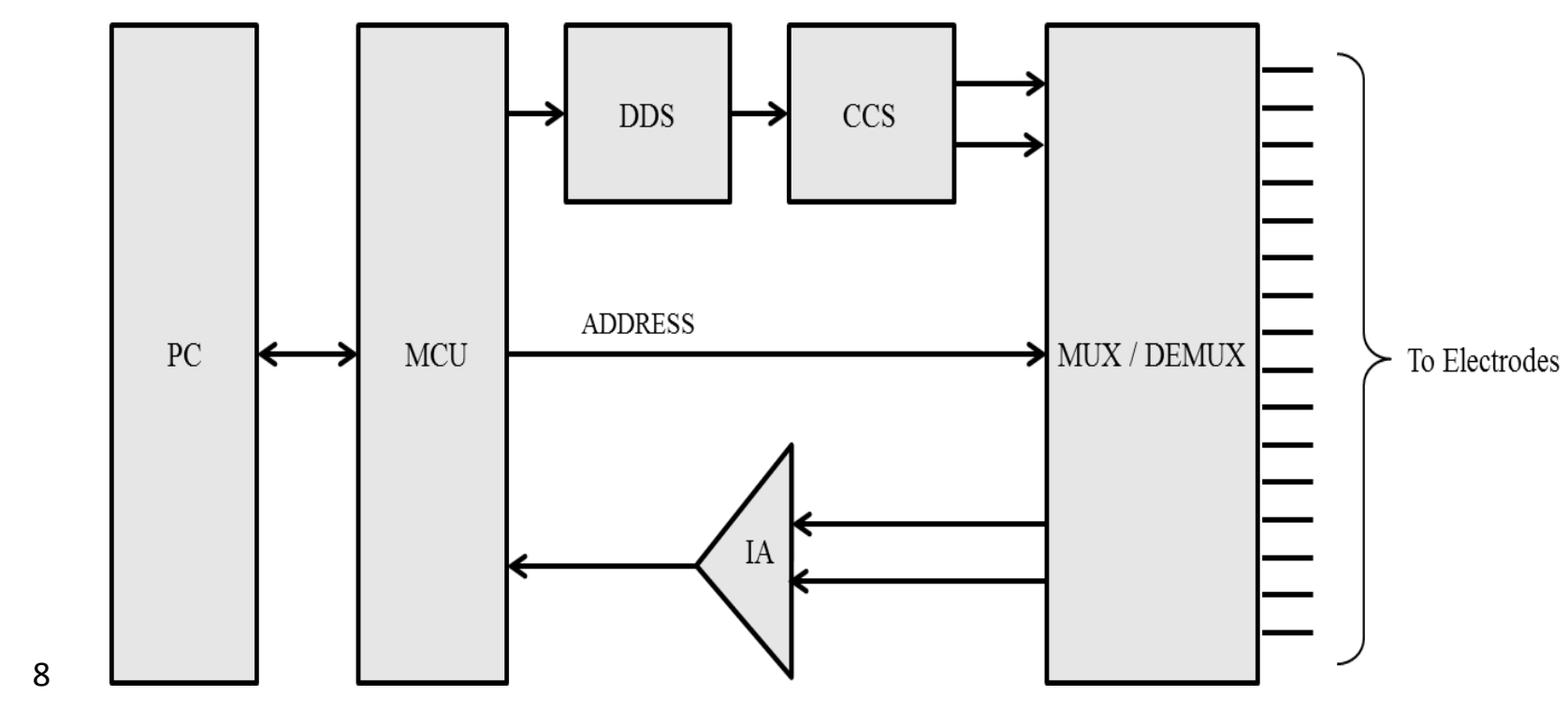

$9 \quad$ Figure 2: EITLboro architectural overview. This system is based on a microcontroller connected 10 to a PC through a serial port. A graphical user interface was developed using Visual Basic (VB).

11 The Constant Current Source, CCS generates a constant current fed by a signal generated by the 12 Direct Digital Synthesizer, DDS. The measurements were amplified using an Instrumentation Amplifier (IA) to produce a complete voltage data set. 


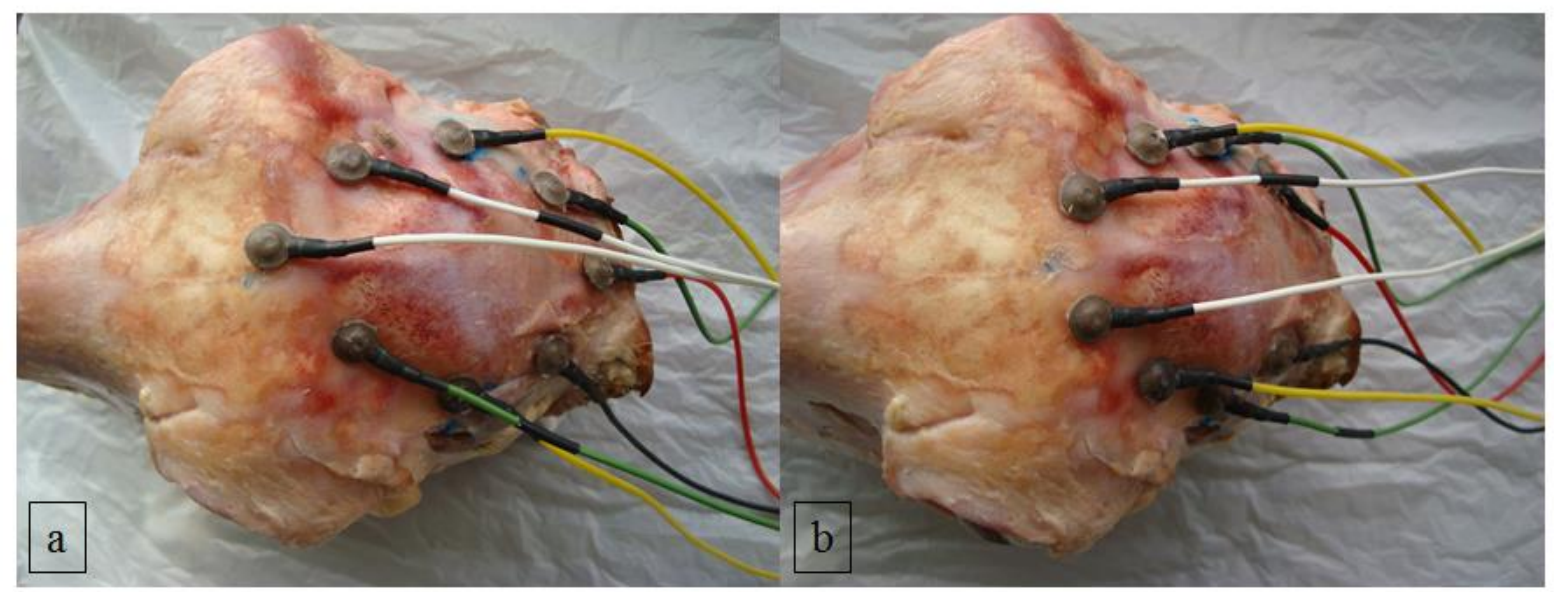

Figure 3: Electrode positions showing (a) eight $\mathrm{Ag} / \mathrm{AgCl}$ disk electrodes fastened to the skull using conductive paste for full array layout and (b) electrodes connected to the skull on the interior of the head using conductive paste for semi-array layout. 


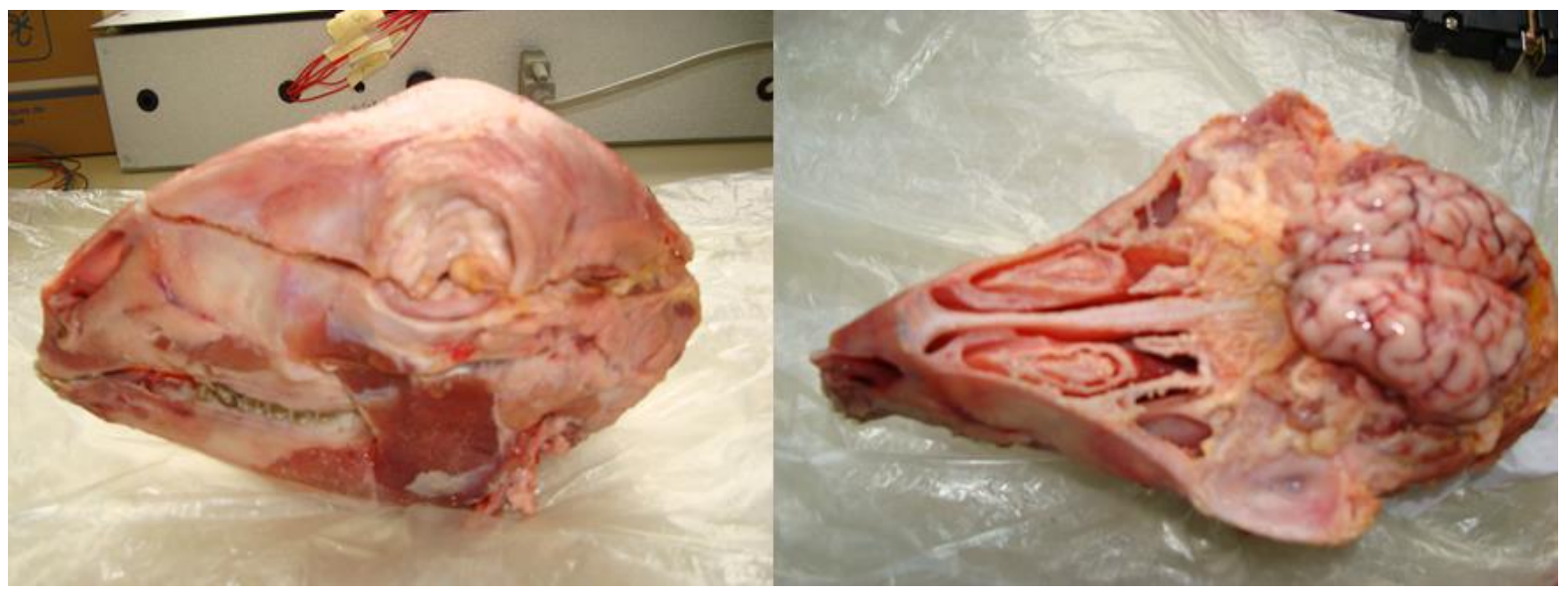

$19 \quad$ Figure 4: Skulls were cut in approximately half using a bone saw (left). The top half of the skull was carefully removed and the brain was exposed to place the anomalies (right). 


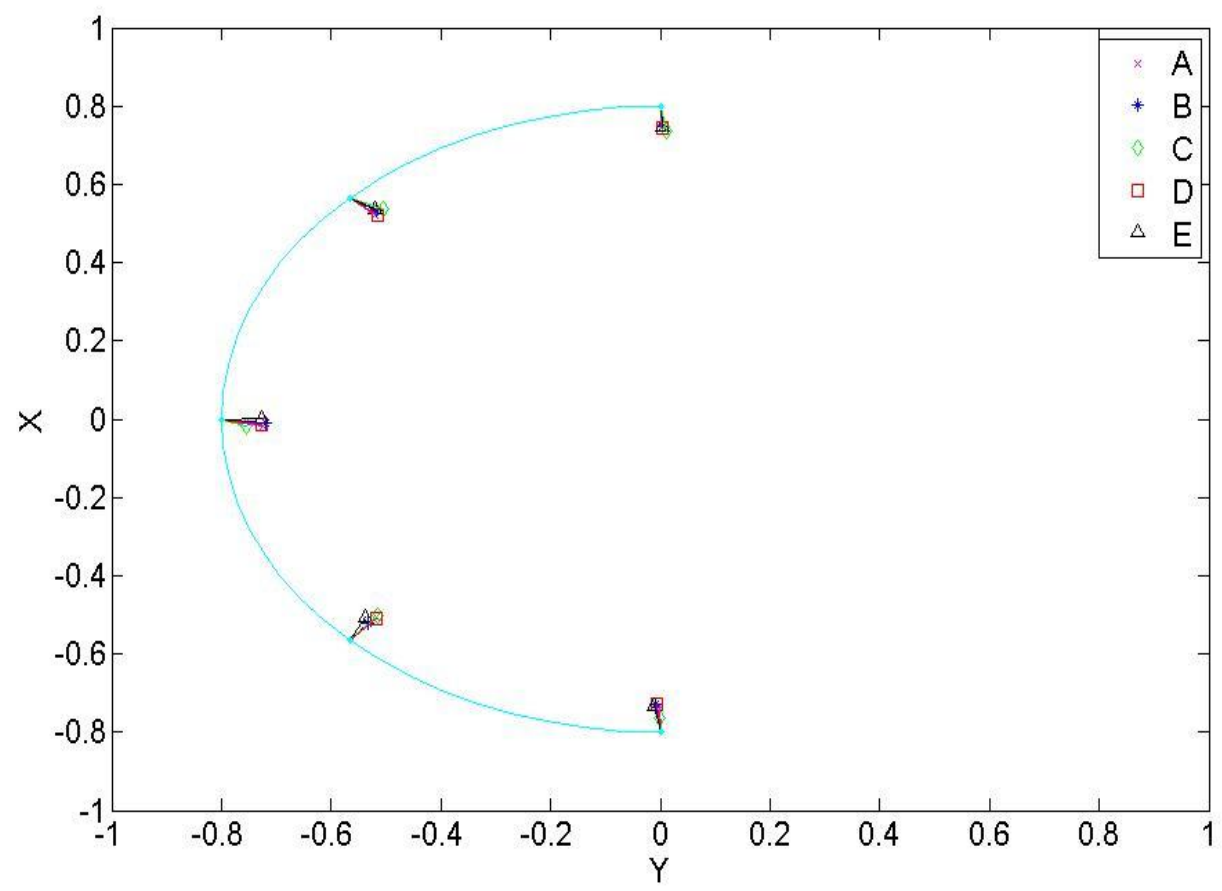

21

(a)

22

23

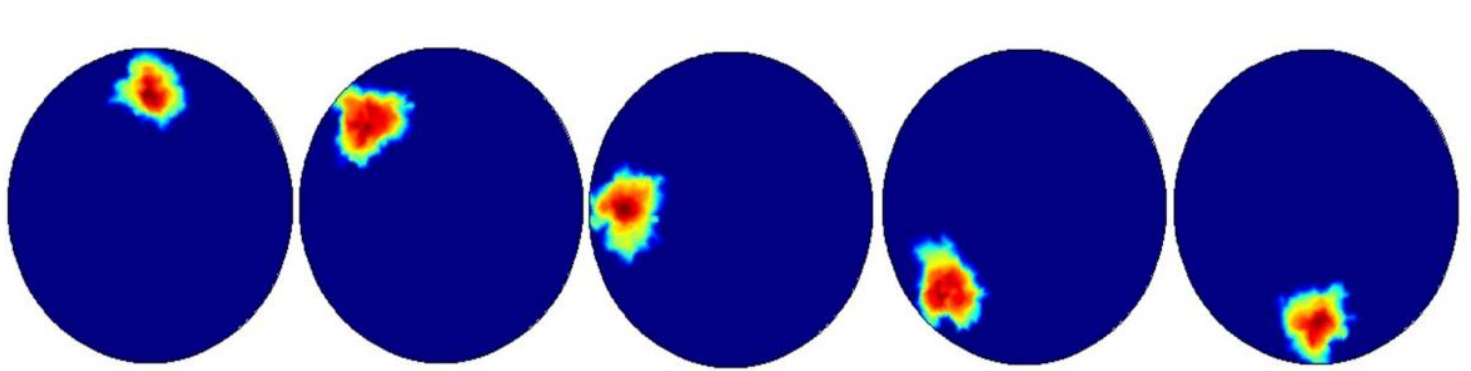

(b)

Figure 5: (a) Localization of the simulated anomalies on five sheep's head (A, B, C, D and E) at various positions using full array (FA) electrode layout. Anomaly positions varied as a function of angle $\left(0^{\circ}, 45^{\circ}, 90^{\circ}, 135^{\circ}\right.$ and $\left.180^{\circ}\right)$ at relative radius of 0.8.

(b) Reconstructed images of the anomalies in subject $\mathrm{E}$. 


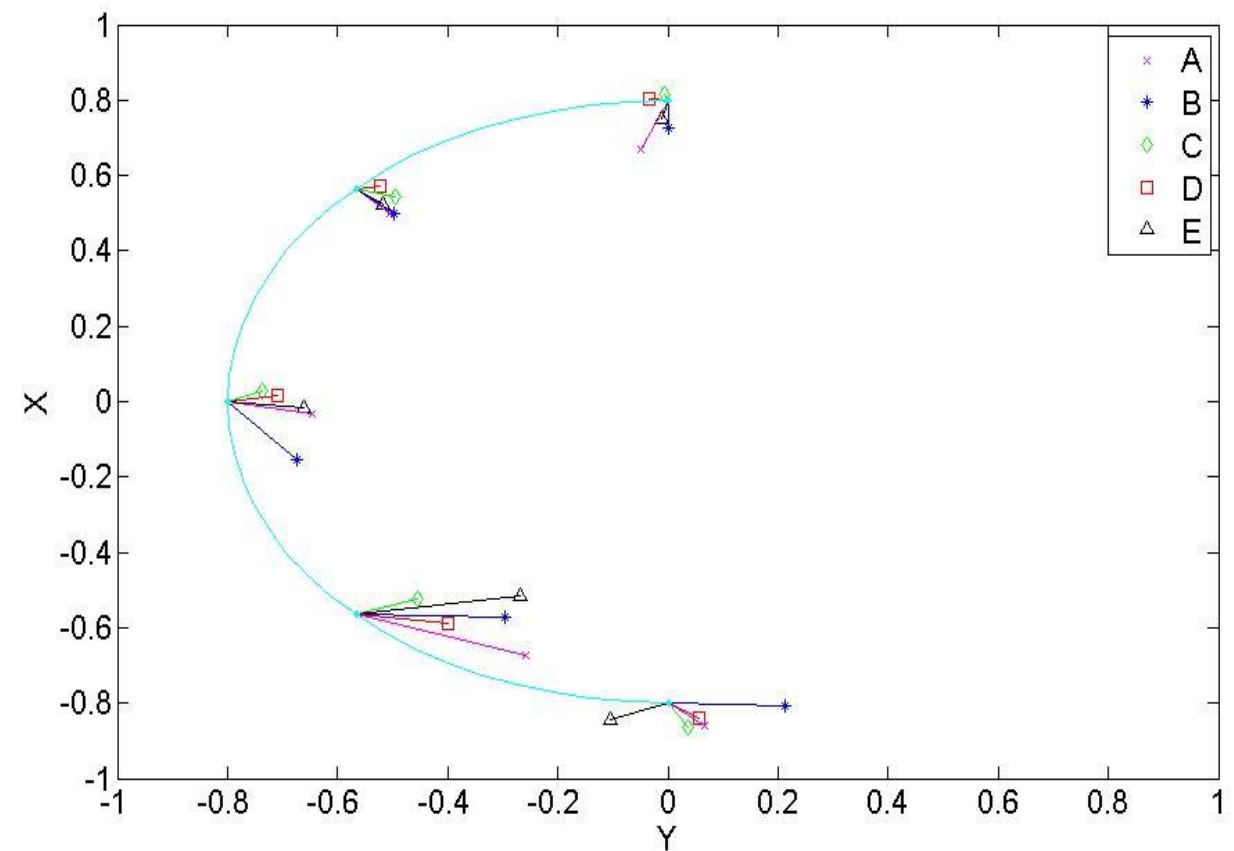

28

(a)
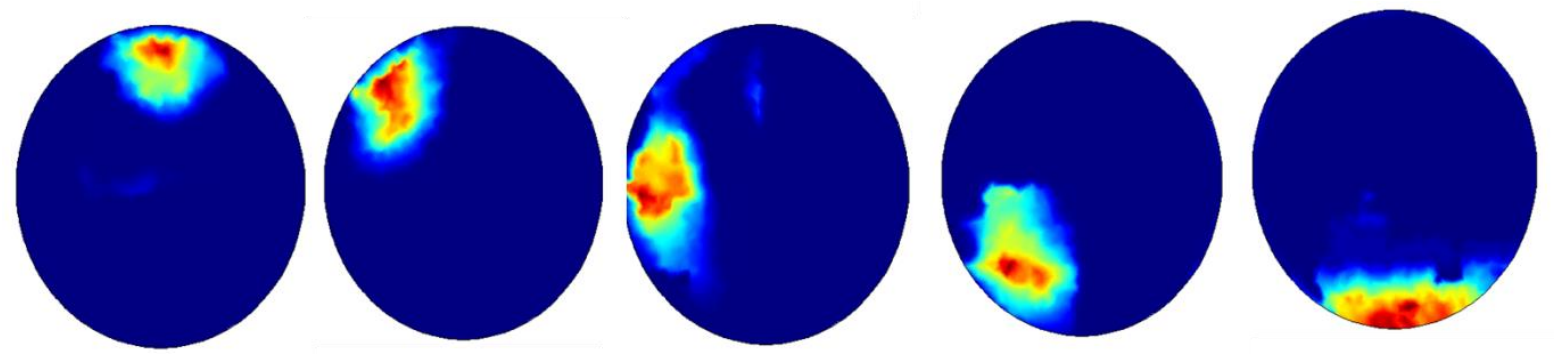

(b)

Figure 6: (a) Localization of the simulated anomalies on five sheep's head (A, B, C, D and E) at various positions using semi-array (SA) electrode layout. Anomaly positions varied as a function of angle $\left(0^{\circ}, 45^{\circ}, 90^{\circ}, 135^{\circ}\right.$ and $\left.180^{\circ}\right)$ at relative radius of 0.8 .

(b) Reconstructed images of the anomalies in subject $\mathrm{C}$. 


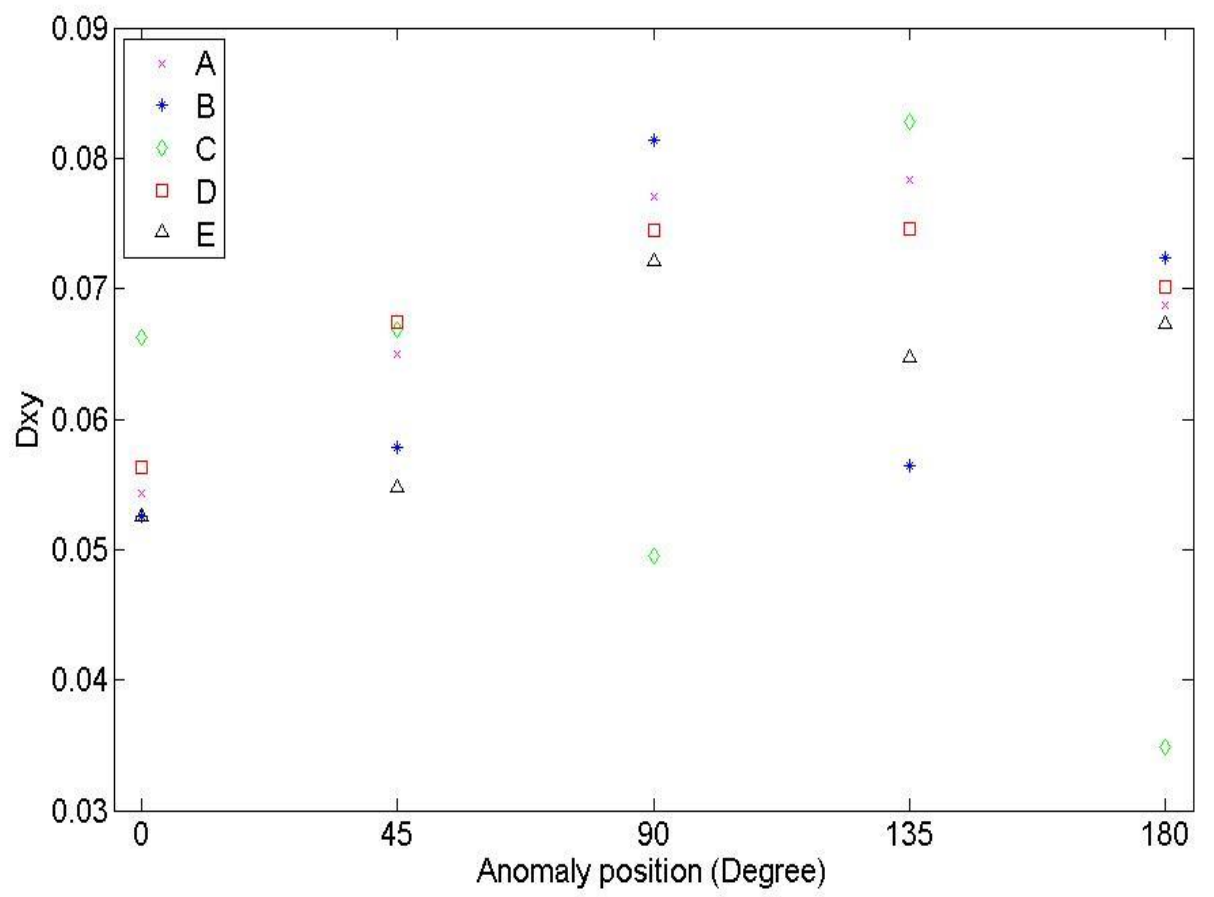

36 Figure 7: Relative radius localization errors, Dxy, of the simulated anomalies on five sheep's

37 head (A, B, C, D and E) at various positions using full array (FA) electrode layout. Anomaly 38 positions varied as a function of angle $\left(0^{\circ}, 45^{\circ}, 90^{\circ}, 135^{\circ}\right.$ and $\left.180^{\circ}\right)$ at relative radius of 0.8 . 


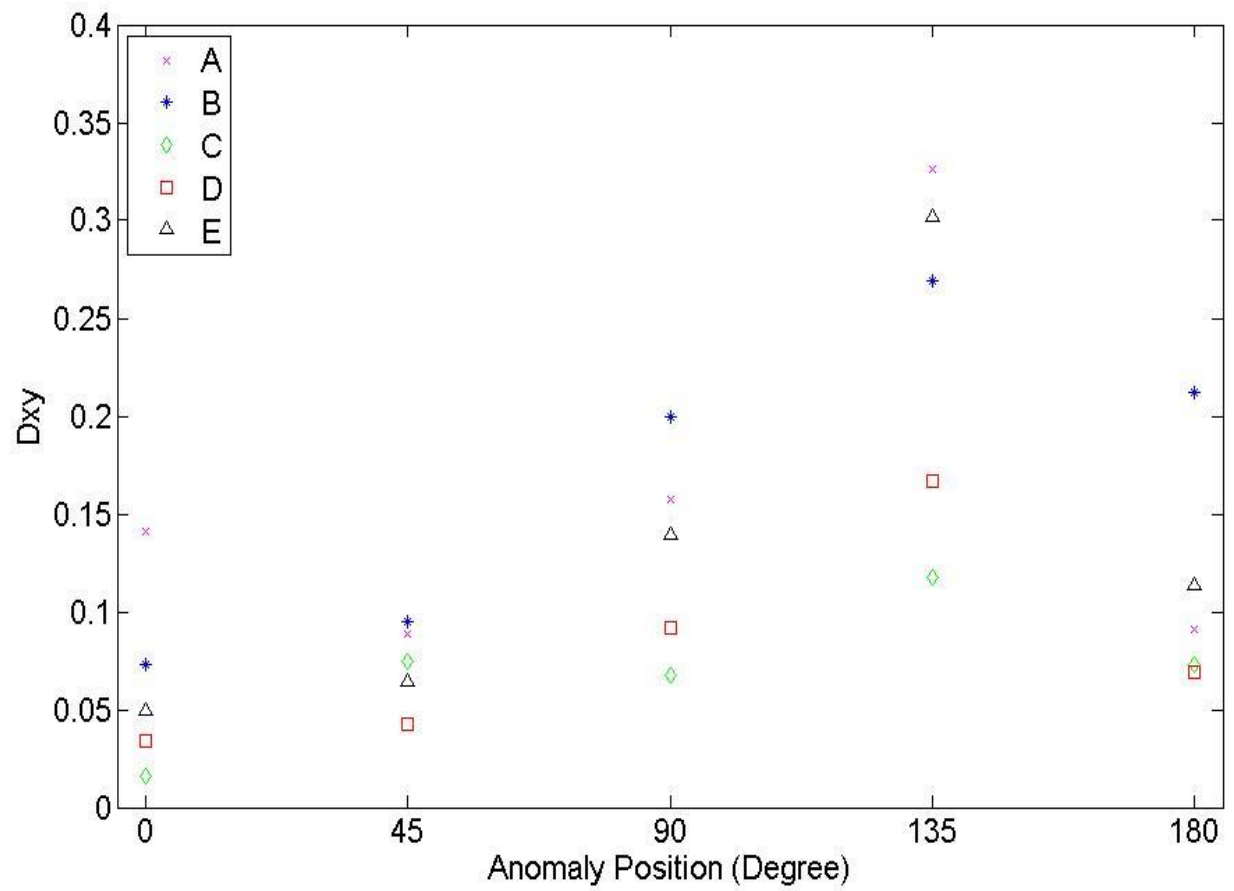

$40 \quad$ Figure 8: Radius relative localization errors, Dxy, of the simulated anomalies on five sheep's

41 head (A, B, C, D and E) at various positions using semi-array (SA) electrode layout. Anomaly positions varied as a function of angle $\left(0^{\circ}, 45^{\circ}, 90^{\circ}, 135^{\circ}\right.$ and $\left.180^{\circ}\right)$ at relative radius of 0.8 . 


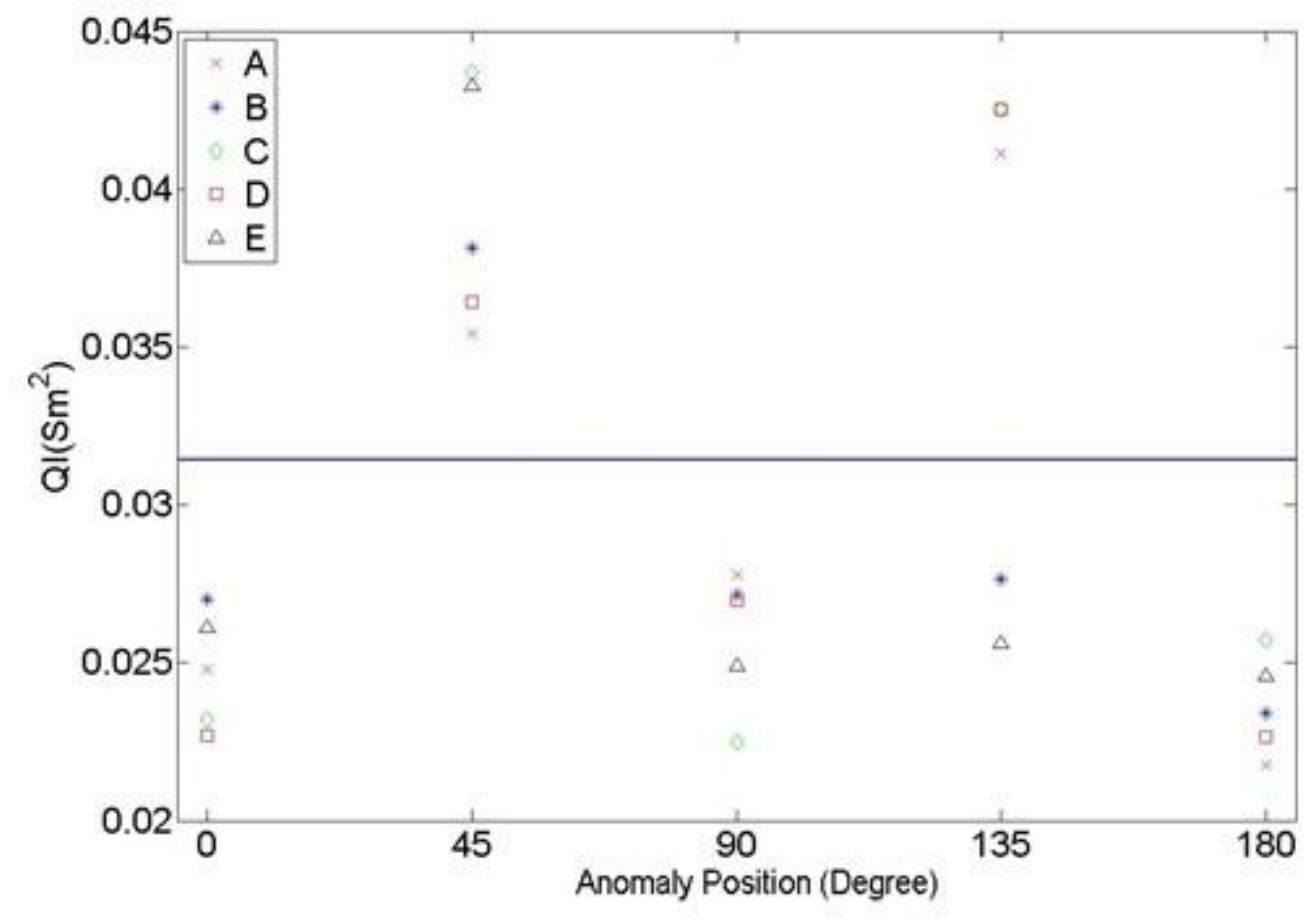

43

$44 \quad$ Figure 9: Quantification Indices, QI, of the simulated anomalies on five sheep's head (A, B, C, D

45 and E) at various positions using full array (FA) electrode layout compared with ideal QI.

46 Anomaly positions varied as a function of angle $\left(0^{\circ}, 45^{\circ}, 90^{\circ}, 135^{\circ}\right.$ and $\left.180^{\circ}\right)$ at relative radius of

47 0.8 . 


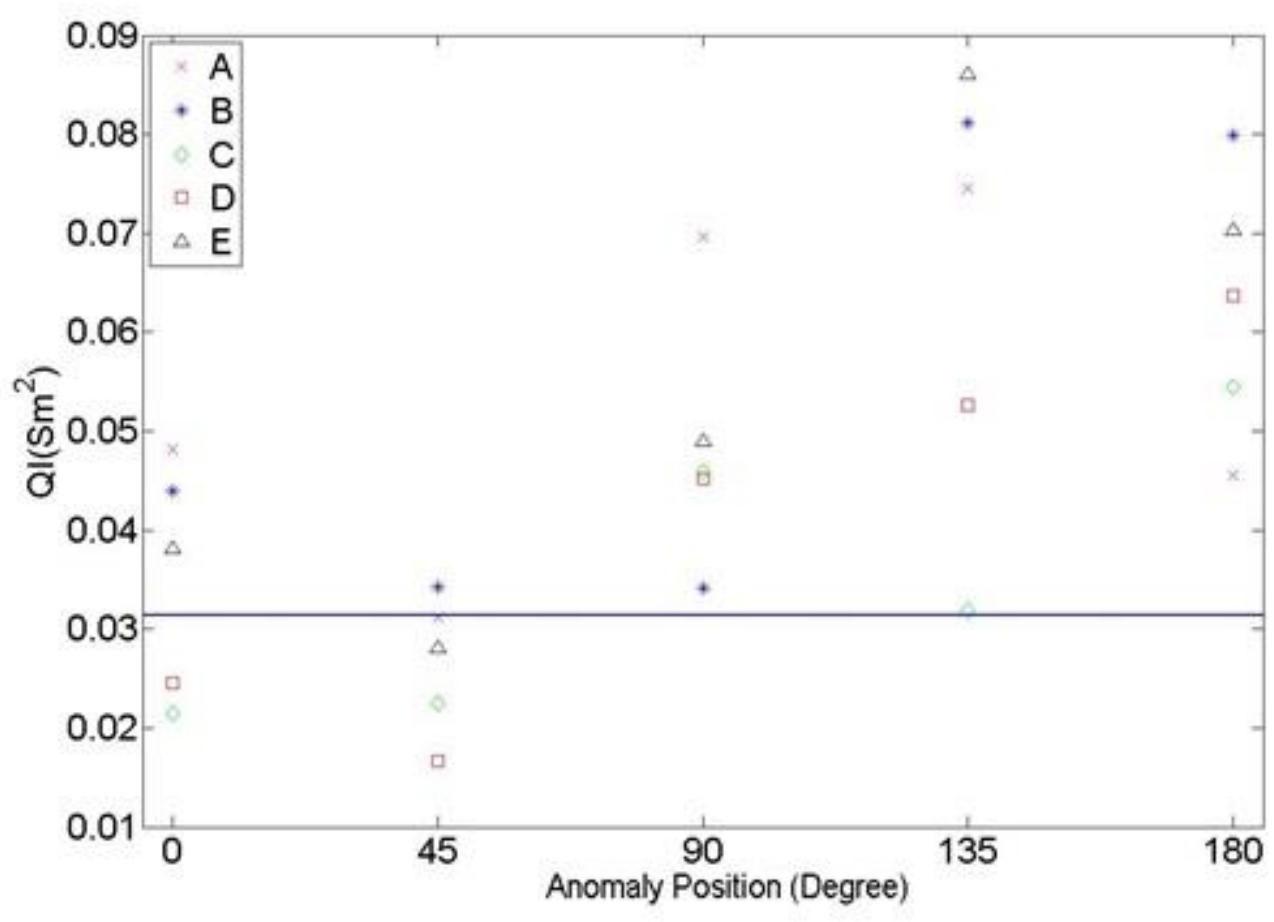

Figure 10: Quantification Indices, QI, of the simulated anomalies on five sheep's head (A, B, C,

50 D and E) at various positions using semi-array (SA) electrode layout compared with ideal QI.

51 Anomaly positions varied as a function of angle $\left(0^{\circ}, 45^{\circ}, 90^{\circ}, 135^{\circ}\right.$ and $\left.180^{\circ}\right)$ at relative radius of

52 0.8 . 